\title{
Pressure distribution of the high-redshift cluster of galaxies CL J1226.9+3332 with NIKA ${ }^{\star}$
}

\author{
R. Adam ${ }^{1}$, B. Comis ${ }^{1}$, J.-F. Macías-Pérez ${ }^{1}$, A. Adane ${ }^{2}$, P. Ade ${ }^{3}$, P. André ${ }^{4}$, A. Beelen ${ }^{5}$, B. Belier ${ }^{6}$, A. Benoît $^{7}$,
} A. Bideaud ${ }^{3}$, N. Billot ${ }^{8}$, G. Blanquer ${ }^{1}$, O. Bourrion ${ }^{1}$, M. Calvo ${ }^{7}$, A. Catalano ${ }^{1}$, G. Coiffard ${ }^{2}$, A. Cruciani ${ }^{15}$, A. D’ Addabbo ${ }^{7,15}$, F.-X. Désert ${ }^{9,10}$, S. Doyle ${ }^{3}$, J. Goupy ${ }^{7}$, C. Kramer ${ }^{8}$, S. Leclercq ${ }^{2}$, J. Martino ${ }^{5}$, P. Mauskopf ${ }^{3,14}$, F. Mayet ${ }^{1}$, A. Monfardini ${ }^{7}$, F. Pajot ${ }^{5}$, E. Pascale ${ }^{3}$, L. Perotto ${ }^{1}$, E. Pointecouteau ${ }^{11,12}$, N. Ponthieu ${ }^{9,10}$, V. Revéret ${ }^{4}$, A. Ritacco ${ }^{1}$, L. Rodriguez ${ }^{4}$, G. Savini ${ }^{13}$, K. Schuster ${ }^{2}$, A. Sievers ${ }^{8}$, C. Tucker ${ }^{3}$, and R. Zylka ${ }^{2}$

1 Laboratoire de Physique Subatomique et de Cosmologie (LPSC), Université Grenoble-Alpes, CNRS/IN2P3, 53 avenue des Martyrs, 38026 Grenoble, France e-mail: adam@lpsc.in2p3.fr

2 Institut de RadioAstronomie Millimétrique (IRAM), 38406 Grenoble, France

3 Astronomy Instrumentation Group, University of Cardiff, UK

${ }^{4}$ Laboratoire AIM, CEA/IRFU, CNRS/INSU, Université Paris Diderot, CEA-Saclay, 91191 Gif-Sur-Yvette, France

5 Institut d'Astrophysique Spatiale (IAS), CNRS and Université Paris Sud, 91405 Orsay, France

${ }^{6}$ Institut d'Electronique Fondamentale (IEF), Université Paris Sud, 91405 Orsay, France

7 Institut Néel, CNRS and Université de Grenoble, 38000 Grenoble, France

8 Institut de RadioAstronomie Millimétrique (IRAM), Granada, Spain

9 Univ. Grenoble Alpes, IPAG, 38000 Grenoble, France

${ }^{10}$ CNRS, IPAG, 38000 Grenoble, France

11 Université de Toulouse, UPS-OMP, Institut de Recherche en Astrophysique et Planétologie (IRAP), Toulouse, France

12 CNRS, IRAP, 9 Av. colonel Roche, BP 44346, 31028 Toulouse Cedex 4, France

13 University College London, Department of Physics and Astronomy, Gower Street, London WC1E 6BT, UK

14 School of Earth and Space Exploration and Department of Physics, Arizona State University, Tempe, AZ 85287, USA

15 Dipartimento di Fisica, Sapienza Università di Roma, Piazzale Aldo Moro 5, 00185 Roma, Italy

Received 10 October 2014 / Accepted 28 November 2014

\section{ABSTRACT}

\begin{abstract}
The thermal Sunyaev-Zel'dovich (tSZ) effect is expected to provide a low scatter mass proxy for galaxy clusters since it is directly proportional to the cluster thermal energy. The tSZ observations have proven to be a powerful tool for detecting and studying them, but high angular resolution observations are now needed to push their investigation to a higher redshift. In this paper, we report high angular $(<20 \mathrm{arcsec})$ resolution tSZ observations of the high-redshift cluster CL J1226.9+3332 $(z=0.89)$. It was imaged at 150 and $260 \mathrm{GHz}$ using the NIKA camera at the IRAM 30-m telescope. The $150 \mathrm{GHz}$ map shows that CL J1226.9+3332 is morphologically relaxed on large scales with evidence of a disturbed core, while the $260 \mathrm{GHz}$ channel is used mostly to identify point source contamination. NIKA data are combined with those of Planck and X-ray from Chandra to infer the cluster's radial pressure, density, temperature, and entropy distributions. The total mass profile of the cluster is derived, and we find $M_{500}=5.96_{-0.79}^{+1.02} \times 10^{14} M_{\odot}$ within the radius $R_{500}=930_{-43}^{+50} \mathrm{kpc}$, at a $68 \%$ confidence level. $\left(R_{500}\right.$ is the radius within which the average density is 500 times the critical density at the cluster's redshift.) NIKA is the prototype camera of NIKA2, a KIDs (kinetic inductance detectors) based instrument to be installed at the end of 2015. This work is, therefore, part of a pilot study aiming at optimizing tSZ NIKA2 large programs.
\end{abstract}

Key words. techniques: high angular resolution - galaxies: clusters: individual: CL J1226.9+3332

\section{Introduction}

Galaxy clusters are the largest gravitationally bound objects in the Universe. They arise from the collapse of primordial matter fluctuations, forming overdensity peaks at the intersection of filamentary structures. They offer a unique tracer of the matter distribution and a powerful probe for cosmology because they form across the expansion of the Universe. (See, for example, Allen et al. 2011 and references therein for a detailed review.)

* The FITS file of the published maps is only available at the CDS via anonymous ftp to cdsarc.u-strasbg. fr (130.79.128.5) or via http://cdsarc.u-strasbg.fr/viz-bin/qcat?J/A+A/576/A12
Clusters are mainly made of dark matter (about $85 \%$ of their total masses), but also of hot ionized gas (about 12\%) and of the stars and interstellar medium within galaxies (a few percent), representing the baryonic component that can be used to detect and study them. Optical observations have been historically used to measure their total mass (Zwicky 1933) - typically around $10^{14} M_{\odot}$ - from galaxy velocity dispersion and, more recently, from lensing measurements of background objects (see Bartelmann 2010, for a review). Since galaxy clusters form by accreting of surrounding material (dark matter, galaxies, and gas) and by merging with other clusters, they can be the source of a significant amount of non-thermal emission. Radio measurements around $1 \mathrm{GHz}$ are used to explore such processes 
(e.g., Feretti et al. 2011). The hot gas contained in the intracluster medium (ICM) - a few $\mathrm{keV}$ - emits X-ray photons due to the bremsstrahlung of energetic electrons (see Sarazin 1988). Therefore, X-ray imaging can be used to study the electronic density distribution in galaxy clusters (with a weak dependence on the temperature). In addition, X-ray spectroscopy provides the possibility of measuring the ICM temperature (see, for example, Böhringer \& Werner 2010).

To be used for cosmology, galaxy clusters observables need to be related in some way to their total mass. The precise calibration of such scaling relations requires to use as many available probes (that are complementary to each other) as possible. The thermal Sunyaev-Zel'dovich (tSZ, Sunyaev \& Zel'dovich 1972, 1980) effect provides such a probe. It is due to the inverse Compton scattering of cosmic microwave background (CMB) photons with high-energy electrons in the ICM. The photons are shifted to higher frequencies providing a characteristic spectral distortion of the CMB, observable at millimeter wavelengths. Since the observable is not the cluster itself but the CMB, tSZ offers a key advantage because it does not suffer from cosmological dimming as do other probes. Its amplitude is directly proportional to the pressure distribution in clusters and is therefore expected to provide a low scatter mass proxy when assuming hydrostatic equilibrium (e.g., Nagai 2006). Together with X-ray observations, the tSZ effect allows for a detailed characterization of the ICM thermodynamics. See Birkinshaw (1999), Carlstrom et al. (2002), and Kitayama (2014) for detailed reviews on the tSZ effect.

The Planck satellite (Planck Collaboration XXIX 2014), the South Pole Telescope (SPT, Reichardt et al. 2013; Bleem et al. 2015), and the Atacama Cosmology Telescope (ACT, Hasselfield et al. 2013) have produced, and will continue to improve, large tSZ selected cluster samples. However, as the highredshift end of these samples is reached, clusters are not resolved owing to insufficient available angular resolution (larger than 1 arcmin). High angular resolution follow-up observations of these objects are needed to precisely calibrate the tSZ cluster observable versus their total mass, through their pressure profiles. The universality of such pressure distributions (Planck Collaboration Int. V 2013; Arnaud et al. 2010), taken as a standard candle, also has to be tested against redshift.

The object CL J1226.9+3332 is a high-redshift, hot and massive cluster of galaxies at $z=0.89$. It was discovered in the WARPS survey (Wide Angle ROSAT Pointed Survey, Ebeling et al. 2001) and has been the object of multiwavelength studies. Owing to difficulty of X-ray spectroscopy at highredshift, the first temperature estimates were made from SZA (Sunyaev-Zel'dovich Array) observations (Joy et al. 2001, $T_{\mathrm{e}}=$ $9.8_{-1.9}^{+4.7} \mathrm{keV}$ ), providing the first confirmation that it is indeed a massive system. A detailed X-ray analysis of XMM-Newton observations by Maughan et al. (2004) reports a consistent temperature, $T_{\mathrm{e}}=11.5_{-0.9}^{+1.1} \mathrm{keV}$. They also measured CL J1226.9+3332 to show evidence of a relaxed X-ray morphology, in agreement with ROSAT first observations, and provided a total mass of $(1.4 \pm 0.5) \times 10^{15} M_{\odot}$. More recent Chandra observations also agree that CL J1226.9+3332 is a hot system (Bonamente et al. $\left.2006, T_{\mathrm{e}}=14.0_{-1.8}^{+2.1} \mathrm{keV}\right)$. The pressure profile of the cluster was measured at arcmin angular scales using the interferometric SZA observations at 30 and $90 \mathrm{GHz}$ (Muchovej et al. 2007; Mroczkowski et al. 2009; Mroczkowski 2011), providing a detailed picture of the ICM on these scales. First indications of a disturbed core were made by an XMM/Chandra analysis, showing an asymmetry in the temperature map with a hotter southwest region (Maughan et al. 2007). Lensing observations by the
Hubble Space Telescope (HST Jee \& Tyson 2009) found a relaxed morphology on large scales and agree on the presence of a disturbed core on smaller scales, with the presence of a subclump 40 arcsec toward the southwest. This is consistent with the hotter region and highly correlated with the cluster galaxy distribution. They propose a scenario in which a less massive system has passed through the main cluster and the gas has been stripped during this passage. The mass inferred within the radius $^{1} r_{\Delta=200}$ is $(1.4 \pm 0.2) \times 10^{15} M_{\odot}$. Finally, MUSTANG tSZ observations at $90 \mathrm{GHz}$ on $\sim 8-45$ arcsec scales (Korngut et al. 2011) have revealed a narrow 20 arcsec long ridge 10 arcsec southwest of the X-ray peak, in addition to another peak coincident with X-ray and the brightest cluster galaxy.

In this paper, we report 150 and $260 \mathrm{GHz}$ tSZ observations of CL J1226.9+3332, using the New IRAM KIDs Array (NIKA, see Monfardini et al. 2010, 2011; Bourrion et al. 2011; Calvo et al. 2013; Adam et al. 2014; Catalano et al. 2014, for more details on the NIKA camera) at the IRAM (Institut de Radio Astronomie Millimétrique) 30-m telescope. The reconstructed tSZ map of the cluster is used to constrain its pressure distribution, as well as the thermodynamics of the ICM gas by combining it with X-ray data. Since NIKA is the prototype of the future NIKA2 camera, these observations are part of a pilot study that aims at showing the potential of NIKA2 for follow-ups of unresolved Planck and ACT clusters.

The paper is organized as follows. The observations of CL J1226.9+3332 are presented in Sect. 2, including the reduction of NIKA data. In Sect. 3, we present the analysis performed to recover the thermodynamical properties of the cluster. The results are provided in Sect. 4 and compared to data of other observatories and previous observations. The conclusions and NIKA2 perspectives are given in Sect. 5. Throughout this paper we assume a flat $\Lambda \mathrm{CDM}$ cosmology according to the latest Planck results (Planck Collaboration XVI 2014) with $H_{0}=67.11 \mathrm{~km} \mathrm{~s}^{-1} \mathrm{Mpc}^{-1}, \Omega_{\mathrm{M}}=0.3175$, and $\Omega_{\Lambda}=0.6825$.

\section{High resolution thermal Sunyaev-Zel'dovich observations}

\subsection{Observations of CL J1226.9+3332}

The NIKA camera was used at the IRAM 30-m telescope (Pico Veleta, Spain) to image CL J1226.9+3332 at 150 and $260 \mathrm{GHz}$ during the first NIKA open pool of February 2014. The cluster was mapped using on-the-fly raster scans made of constant azimuth - resp. elevation - subscans. Scans were made of 19 subscans of 6 arcmin length, separated by 10 arcsec elevation - resp. azimuth - steps. The subscan duration was fixed to ten seconds, giving a scanning speed of 36 arcsec per second and a total time of 3.3 minutes per scan. The pointing center was chosen to be

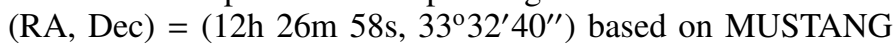
tSZ observations (Korngut et al. 2011). All coordinates in this paper are given in equinox 2000.

The data collected were taken with an opacity, at $150 \mathrm{GHz}$ (respectively $260 \mathrm{GHz}$ ), in the range $0.06-0.23$ (respectively $0.06-0.29$ ) and a mean value of 0.13 (respectively 0.16 ), corresponding to average winter conditions. The observations were mostly carried out during night time. A small fraction of the scans were flagged due to bad weather conditions and some others were lost because of missing data streams with the telescope

1 The radius $r_{\Delta}$ corresponds to a radius within which the mean cluster density is $\Delta$ times the one of the critical density of the Universe at that redshift. Hereafter, we commonly use the physical quantities within this radius, noted with a subscript $\Delta$ generally taken as 500 . 
Table 1. Instrumental characteristics of NIKA for the February 2014 campaign.

\begin{tabular}{ccc}
\hline \hline Observing band & $150 \mathrm{GHz}$ & $260 \mathrm{GHz}$ \\
\hline Gaussian beam model FWHM (arcsec) & 18.2 & 12.0 \\
Field-of-view (arcmin) & 1.9 & 1.8 \\
Effective number of detectors & 117 & 136 \\
Sensitivity (mJy/beam s s/2) & 14 & 35 \\
Compton parameter to Jy/beam & $-10.9 \pm 0.8$ & $3.5 \pm 0.5$ \\
Pointing errors (arcsec) & $<3$ & $<3$ \\
Calibration uncertainties & $7 \%$ & $12 \%$ \\
\hline
\end{tabular}

Notes. See text for details.

position. The overall effective observing time on the cluster is $7.8 \mathrm{~h}$.

The overall final pointing residual errors were obtained with a precision of less than 3 arcsec using the observations of nearby quasars, $1308+326$ and $1156+295$, every hour. Uranus was taken as our primary calibrator, and we used its frequencydependent brightness temperature model as given by Moreno (2010), assumed to be accurate at the level of 5\%, as shown by Planck Collaboration VIII (2014). Within the NIKA bandpasses of the February 2014 campaign, we obtain a mean brightness temperature of 112.7 and $92.8 \mathrm{~K}$ at 150 and $260 \mathrm{GHz}$, respectively. Uncertainties on the calibration were measured to be $5 \%$ and $11 \%$ at 150 and $260 \mathrm{GHz}$, respectively, using the dispersion of the recovered flux on Uranus maps. This corresponds to $7 \%$ and $12 \%$ overall calibration uncertainties when including the model error. The focus of the telescope was checked on Uranus or other bright point sources every two to three hours and systematically after sunset and sunrise. The effective FWHM was measured to be 18.2 and 12.0 arcsec at 150 and $260 \mathrm{GHz}$, respectively, by fitting a Gaussian model on the planet. The opacity was measured and corrected for by using NIKA total power data as a tau-meter as described in Catalano et al. (2014). The Compton $y$ to surface brightness (measured in Jy/beam) conversions were computed by integrating the tSZ spectrum (see Sect. 3) within the NIKA bandpasses transmissions and accounting for the beam angular coverage, as described in more detail in Adam et al. (2014). For this campaign, the conversions are $-10.9 \pm 0.8$ and $3.5 \pm 0.5 \mathrm{Jy} /$ beam per unit of $y$ at 150 and $260 \mathrm{GHz}$, respectively, including the overall calibration error and the $2 \%$ error arising from the bandpasses uncertainties.

In Table 1, we summarize the instrumental properties of the NIKA camera as it was used during CL J1226.9+332 observation.

\subsection{Data reduction}

The details of the NIKA data reduction are available in Adam et al. (2014) and Catalano et al. (2014). Here, the main procedure is briefly summarized for the reader's convenience. Invalid detectors were removed based on the statistical properties of their noise and their optical response. Cosmic ray impacts on the arrays were flagged and removed from the data. To remove the low-frequency atmospheric emission from the data, a common-mode template was built by averaging the detector time stream across each array. This was done by flagging the source in signal-to-noise in an iterative manner to avoid ringing and reduce signal filtering effects. This data reduction was preferred for CL J1226.9+3332, with respect to the spectral dualband noise decorrelation described in Adam et al. (2014). The latter allows more extended emission to be recovered but is noisier. Moreover, the cluster is sufficiently compact for any filtering effect to be weak enough, allowing the recovery of cluster maps at both wavelengths simultaneously. Frequency lines produced by the pulse tube of the cryostat were notch-filtered in the Fourier domain. Data were finally projected onto 2-arcsec pixel grid maps using inverse variance weighting and a nearest grid projection.

The raw (direct output of the pipeline) 150 and $260 \mathrm{GHz}$ NIKA maps of CL J1226.9+3332 are presented in Fig. 1. They are centered on the X-ray peak coordinates (taken from Cavagnolo et al. 2009), (RA, Dec) =

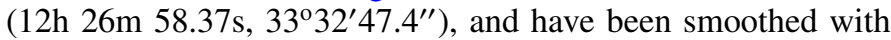
a 10 arcsec Gaussian filter for display purposes. The $150 \mathrm{GHz}$ map shows a strong tSZ decrement reaching $18 \sigma$ per beam at the peak. Because the noise is higher at $260 \mathrm{GHz}$, and the tSZ signal weaker by a factor of about one third, the map does not show a very significant $\mathrm{tSZ}$ detection, even if diffuse positive emission is seen at about $3 \sigma$ on the map at the cluster position. However, the $260 \mathrm{GHz}$ channel reveals the presence of a point source (referred to as PS260 in the following) located about 30 arcsec southeast of the X-ray center, detected at about $10 \sigma$. The source is not clearly detected at $150 \mathrm{GHz}$ due to the strong tSZ signal, but it is visible as a lack of $\mathrm{tSZ}$ at its corresponding location. In Sect. 2.4, we discuss the implications of point source contamination on our tSZ observation.

In Fig. 2, we provide the flux density profile corresponding to the $150 \mathrm{GHz}$ map. It is computed by averaging the signal in concentric annuli with the X-ray center taken as the origin. The profile appears to be smooth and peaks at the center.

\subsection{Transfer function}

The data reduction described above induces an attenuation of the astrophysical signal in the recovered maps of Fig. 1, since detectors are combined to remove the correlated noise. The characterization of this effective transfer function as a function of the angular scales was done by using noise plus input signal simulations. To do so, the map of an input known simulated astrophysical signal (see below) was compared to the output signal after processing.

The simulated input signal was the one expected for clusters of galaxies, as described in detail in Adam et al. (2014). It was computed using a generalized Navarro, Frenk \& White (gNFW) pressure profile (see Sect. 3.2.1, Nagai et al. 2007b) integrated along the line of sight to produce a tSZ flux density map. The typical amplitude and angular size of the simulated clusters were similar to the one in Fig. 1, but the result transfer function was checked to see that it did not depend on the radial size and amplitude of the input signal.

To simulate the atmospheric and intrinsic correlated and uncorrelated noise, we used the NIKA data themselves. The real data used for the simulations were those of our other projects taken during the first NIKA open pool of February 2014, for which the scanning strategy was similar to that of CL J1226.9+3332. These scans were taken with atmospheric conditions comparable to those during which the data presented in this paper were taken. The astrophysical signal within the data was checked to be faint enough that it did not affect the reduction, i.e. negligible compared to the noise.

To deal with the residual noise contribution in the final processed maps, we considered the simulated data with and without including the known input signal. In this way we obtained estimates of both the processed noisy signal and of the noise itself, which we could subtract from the processed signal-plus-noise 

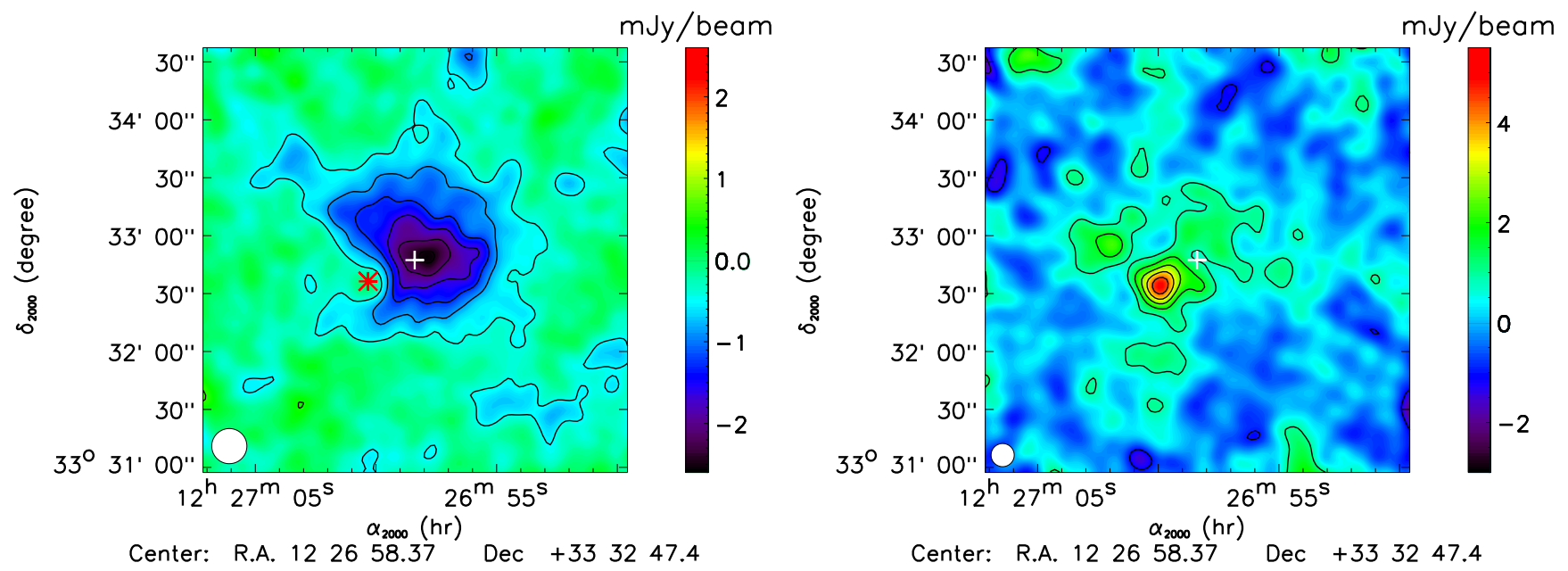

Fig. 1. NIKA raw maps of CL J1226.9+3332 at $150 \mathrm{GHz}$ (left) and $260 \mathrm{GHz}$ (right). Contours are multiples of $3 \sigma$, excluding the zero level, which is not shown. The effective beams FWHM (12.0 and 18.2 arcsec native resolution) are shown as the bottom left white circles, although the display images are smoothed with an extra 10 arcsec FWHM Gaussian. The position of the X-ray center is shown as a white cross in both maps and that of the point source is shown as a red star in the $150 \mathrm{GHz}$ map.

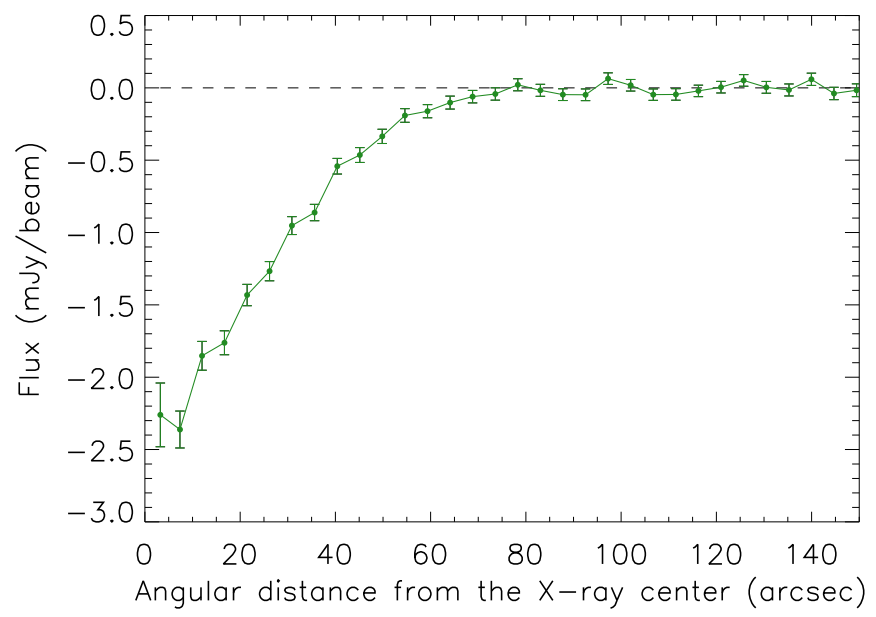

Fig. 2. Flux density profile of CL J1226.9+3332 as measured by NIKA at $150 \mathrm{GHz}$, i.e., the radial average within concentric annuli. The point source has been fitted and subtracted (see Sect. 3.4) before computing the profile. Error bars are only statistical.

map to produce an output signal-only map. The transfer function was then computed as the ratio of the power spectra of the output signal, free of noise, and the input one. However, small noise residuals are observed because of the differences in the processing introduced by the signal itself.

The estimated transfer function is given in Fig. 3. The uncertainties were calculated from the dispersion of the transfer function obtained for all the scans used to compute it, and are mostly due to residual noise. As we can see, it is approximately flat and close to one, with $\sim 5 \%$ attenuation, on scales smaller than the NIKA field of view. On larger scales, the recovered flux vanishes smoothly with decreasing wave number. In Sec. 3.4, we use this transfer function when comparing a model to the NIKA map.

\subsection{Point source contamination}

The SZA data were used to search for radio sources around CL J1226.9+3332 (Muchovej et al. 2007). From their

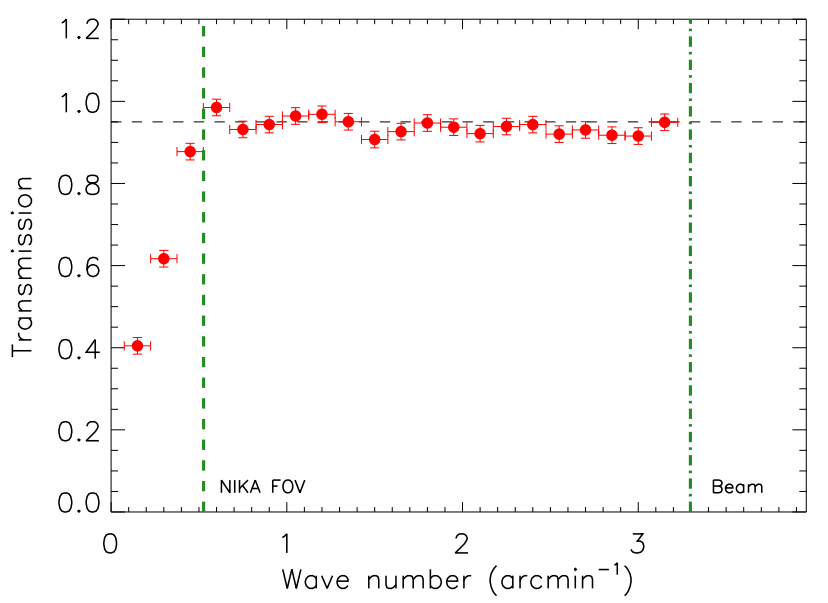

Fig. 3. NIKA data reduction transfer function as a function of angular frequency. Uncertainties were computed using the dispersion of the results over the different noise realizations. The $150 \mathrm{GHz}$ beam cutoff and size of the NIKA field of view are also represented by green dashed lines for illustration, i.e. $(18.2 \mathrm{arcsec})^{-1}$ and $(1.9 \mathrm{arcmin})^{-1}$. The black horizontal dashed line corresponds to $5 \%$ filtering.

observations, no such objects are present within the NIKA field. From the residual between the MUSTANG map and the SZA pressure model of Mroczkowski et al. (2009), Korngut et al. (2011) have inferred the presence of a possible submillimeter source 10 arcsec north of the X-ray peak. Using the NIKA $260 \mathrm{GHz}$ frequency band, we searched for such a contaminant and do not observe any point source within a 1.5 arcmin radius around the map center, apart from PS260. This is done by fitting PS260 and the tSZ signal simultaneously, as described in detail in Sect. 3.4. The root-mean-squared between the best-fit model and the data allows us to set a $2 \sigma$ flux upper limit of $1.5 \mathrm{mJy}$ at this frequency. Therefore, the feature seen by Korngut et al. (2011) is unlikely to be a real submillimeter source. The flux distribution of the detected point source, PS260, is fitted using a Gaussian model with FWHM fixed to the NIKA $260 \mathrm{GHz}$ beam. Its flux is measured to be $6.8 \pm 0.7$ (stat.) \pm 1.0 (cal.) mJy and its position $(\mathrm{RA}$, Dec. $)=\left(12 \mathrm{~h} 27 \mathrm{m0} 01 \mathrm{~s}, 33^{\circ} 32^{\prime} 42.0^{\prime \prime}\right)$, with statistical and calibration error quoted as stat and cal. We note that the source 
possibly coincides with two known optically detected galaxies $\mathrm{J} 12265995+3332405$ and $\mathrm{J} 12265923+3332405$ (Holden et al. 2009). They are located 1.7 and 9.8 arcsec away from the best-fit position obtained, respectively. We conclude that no additional point sources, wether radio or submillimeter, affect the $150 \mathrm{GHz}$ map of NIKA. The detected source is accounted for in the analysis described hereafter.

\section{Characterization of the intracluster medium}

\section{1. $S Z$ and $X$-ray observables}

\subsubsection{Thermal SZ}

The tSZ effect results in a distortion of the CMB black-body spectrum whose frequency dependence is given by (Birkinshaw 1999)

$f\left(x, T_{\mathrm{e}}\right)=\frac{x^{4} \mathrm{e}^{x}}{\left(\mathrm{e}^{x}-1\right)^{2}}\left(x \operatorname{coth}\left(\frac{x}{2}\right)-4\right)\left(1+\delta_{t \mathrm{SZ}}\left(x, T_{\mathrm{e}}\right)\right)$,

where $x=\frac{h v}{k_{\mathrm{B}} T_{\mathrm{CMB}}}$ is the dimensionless frequency, $h$ the Planck constant, $k_{\mathrm{B}}$ the Boltzmann constant, $v$ the observation frequency, and $T_{\mathrm{CMB}}$ the temperature of the $\mathrm{CMB}$. We use the Itoh et al. (1998) relativistic corrections to compute $\delta_{t \mathrm{SZ}}\left(x, T_{\mathrm{e}}\right)$, where $T_{\mathrm{e}}$ is the electrons temperature. The induced change in intensity relative to the primary $\mathrm{CMB}$ intensity $I_{0}$ reads as

$\frac{\Delta I_{t \mathrm{SZ}}}{I_{0}}=y f\left(x, T_{\mathrm{e}}\right)$,

where $y$ is the Compton parameter, which measures the integrated electronic pressure $P_{\mathrm{e}}$ along the line of sight, $\mathrm{d} l$, written as

$y=\frac{\sigma_{\mathrm{T}}}{m_{\mathrm{e}} c^{2}} \int P_{\mathrm{e}} \mathrm{d} l$.

The parameter $\sigma_{\mathrm{T}}$ is the Thomson cross section, $m_{\mathrm{e}}$ is the electron rest mass, and $c$ the speed of light. Neglecting the relativistic corrections, the tSZ spectral distortion is nil around $217 \mathrm{GHz}$, negative below and positive above.

\subsubsection{Kinetic SZ}

In addition to the tSZ effect, the kinetic Sunyaev-Zel'dovich $(\mathrm{kSZ})$ is caused by the motion of the intracluster gas and its electrons relative to the CMB. This motion leads to a Doppler shift of the CMB photons that are scattered via the Compton effect. It can be expressed as (Birkinshaw 1999)

$\frac{\Delta I_{k \mathrm{SZ}}}{I_{0}}=g\left(x, v_{z}, T_{\mathrm{e}}\right) \sigma_{T} \frac{-v_{z}}{c} \int n_{\mathrm{e}} \mathrm{d} l$,

where $v_{z}$ is the line-of-sight peculiar velocity of the cluster with respect to the Hubble flow, which is positive (negative) for a cluster receding from (coming towards) the observer, and $n_{\mathrm{e}}$ the electronic density. The function $g\left(x, v_{z}, T_{\mathrm{e}}\right)$ provides the spectral dependence of the $\mathrm{kSZ}$ effect as

$g\left(x, v_{z}, T_{\mathrm{e}}\right)=\frac{x^{4} \mathrm{e}^{x}}{\left(\mathrm{e}^{x}-1\right)^{2}}\left(1+\delta_{k \mathrm{SZ}}\left(x, v_{z}, T_{\mathrm{e}}\right)\right)$.

Again, we use Itoh et al. (1998) to account for the relativistic corrections $\delta_{k \mathrm{SZ}}$ and neglect the velocity dependance as it is expected to be less than $1 \%$. By writing $\Delta I(v)=A_{t \mathrm{SZ}} \Delta I_{t \mathrm{SZ}}(v)+$ $A_{k \mathrm{SZ}} \Delta I_{k \mathrm{SZ}}(v)$ where $A_{t S Z, k S Z}$ stand for dimensionless amplitudes (see Eqs. (2) and (4)), and assuming the observed region to be isothermal, one can deduce the line-of-sight velocity as $v_{z}=$ $-\frac{A_{k \mathrm{SZ}} k_{\mathrm{B}} T_{\mathrm{e}}}{A_{t \mathrm{SZ}} m_{\mathrm{e}} c}$.
Table 2. Pressure profile parameters for the three models presented in this paper.

\begin{tabular}{cccc}
\hline \hline Model label & $a$ & $b$ & $c$ \\
\hline PPC & 1.33 & 4.13 & 0.014 \\
NNN & 0.9 & 5.0 & 0.4 \\
FPC & free & 4.13 & 0.014 \\
\hline
\end{tabular}

\subsubsection{X-ray emission}

The X-ray surface brightness, in units of counts $\mathrm{cm}^{-2} \mathrm{~s}^{-1} \mathrm{sr}^{-1}$, is related to the electronic density as

$S_{\mathrm{X}}=\frac{1}{4 \pi(1+z)^{4}} \int n_{\mathrm{e}}^{2} \Lambda\left(T_{\mathrm{e}}, Z\right) \mathrm{d} l$.

The parameter $z$ is the redshift, $\Lambda\left(T_{\mathrm{e}}, Z\right)$ is the cooling function that is proportional to $T_{\mathrm{e}}^{1 / 2}$, and $Z$ is the metallicity. Additionally, the gas temperature can be estimated from X-ray spectroscopy.

\subsection{Intracluster medium modeling}

\subsubsection{Pressure profile}

The cluster electronic pressure distribution is modeled by a spherical gNFW profile (Nagai et al. 2007b), described by

$P_{\mathrm{e}}(r)=\frac{P_{0}}{\left(\frac{r}{r_{\mathrm{p}}}\right)^{c}\left(1+\left(\frac{r}{r_{\mathrm{p}}}\right)^{a}\right)^{\frac{b-c}{a}}}$.

The parameter $P_{0}$ is a normalizing constant; $r_{\mathrm{p}}$ is a characteristic radius; and $a, b$, and $c$ set the slopes at intermediate, large, and small radii, respectively. We can also write $P_{0}=P_{\Delta} \times \mathbb{P}_{0}$ and $r_{\Delta}=c_{\Delta} r_{\mathrm{p}}$, where $P_{\Delta}$ is the average pressure within $r_{\Delta}, \mathbb{P}_{0}$ is a normalizing constant, and $c_{\Delta}$ the concentration parameter (Arnaud et al. 2010). The mass enclosed within $r_{\Delta}, M\left(r=r_{\Delta}\right)$, is then related to $P_{\Delta}$ by a scaling law. One can finally define $\theta_{\mathrm{p}, \Delta}=r_{\mathrm{p}, \Delta} / D_{A}$, where $D_{A}$ is the angular distance of the cluster.

In the following, we use three different choices to fix the slope parameters (see Table 2). 1) We fix $c$ to the value obtained by Comis et al. (2011) for this cluster and fix $a$ and $b$ to the one obtained by Planck Collaboration Int. V (2013) when stacking the tSZ signal of 62 nearby clusters. This choice is used as the baseline since the two outer slope parameters have been obtained directly from tSZ data and are expected to provide a good description of most clusters. The parameter $c$ was not fitted by Planck Collaboration Int. V (2013) so we rely on Chandra X-ray data that are specific to CL J1226.9+3332. This set of parameters is referred to PPC in the following. 2) We fix $a, b$, and $c$ to the values obtained by Nagai et al. (2007a) based on X-ray Chandra clusters and numerical simulations. This set of parameters allows us to directly compare our results to that of Mroczkowski et al. (2009), who used them in their modeling. It is referred to as NNN. 3) We fix $b$ and $c$ to values similar to those of PPC, but fit for the parameter $a$ since it corresponds to scales at which NIKA is the most sensitive for this cluster. This choice is referred to as FPC.

The parameters $P_{0}$ and $r_{\mathrm{p}}$ are always allowed to vary. Since the pressure profile parameters are highly degenerate, in particular $a, P_{0}$, and $r_{\mathrm{p}}$, the main difference between the three models relies on the choice of the core slope parameter $c$ and the outer slope parameter $b$, which cannot be constrained directly with the NIKA data. As discussed in Sect. 4, the core slope parameter is 
related to the thermodynamics of the cluster core but does not affect the overall mass determination. By contrast, the outer slope is associated to the steepness of the mass profile at outer radii. Therefore it controls the cluster overall mass at large radii and can lead to a bias in the mass estimate for radii above those constrained directly from the data. By using these three models, we test the impact of the choice of the model in the regions of the ICM profiles for which NIKA is not directly sensitive.

\subsubsection{Density profile}

Following Mroczkowski et al. (2009) and since we use the work of Comis et al. (2011), the electron density profile is described by a simplified version (SVM) of the model suggested by Vikhlinin et al. (2006)

$n_{\mathrm{e}}(r)=n_{\mathrm{e} 0}\left[1+\left(\frac{r}{r_{\mathrm{c}}}\right)^{2}\right]^{-3 \beta / 2}\left[1+\left(\frac{r}{r_{\mathrm{s}}}\right)^{\gamma}\right]^{-\epsilon / 2 \gamma}$,

which is an extension of the $\beta$ model (Cavaliere \& Fusco-Femiano 1978) with an additional steepening freedom at radii larger than $\sim r_{\mathrm{s}}$, for which the slope parameter is $\epsilon$. The core radius is still given by $r_{\mathrm{c}}$, and $\gamma$ accounts for the width of the transition between the two profiles. In the case of $\epsilon=0$, this model is equivalent to the standard $\beta$ model. Similar to Mroczkowski et al. (2009) and Comis et al. (2011), we fix the parameter $\gamma=3$ because it is a good fit to all the clusters considered by Vikhlinin et al. (2006), and leave the other ones as free parameters.

\subsubsection{Temperature and entropy}

Assuming the ideal gas law, the temperature of the electron population can simply be computed as

$k_{\mathrm{B}} T_{\mathrm{e}}(r)=P_{\mathrm{e}}(r) / n_{\mathrm{e}}(r)$.

It is implicitly modeled as the ratio of the distributions given by the gNFW and SVM models of Eqs. (7) and (8). The ICM entropy is defined as (see review from Voit 2005)

$K(r)=\frac{P_{\mathrm{e}}(r)}{n_{\mathrm{e}}(r)^{5 / 3}}$.

\subsubsection{Mass distribution}

When assuming CL J1227.9+3332 to be in hydrostatic equilibrium, its total mass enclosed within $r, M_{\text {tot }}(r)$, is related to the electronic density and pressure profiles through

$$
\frac{\mathrm{d} P_{\mathrm{e}}(r)}{\mathrm{d} r}=-\frac{\mu_{\mathrm{gas}} m_{\mathrm{p}} n_{\mathrm{e}}(r) G M_{\mathrm{tot}}(r)}{r^{2}}
$$

where $m_{\mathrm{p}}$ is the proton mass and $G$ the Newton's constant. We assume in this paper a mean molecular weight $\mu_{\mathrm{e}}=1.15$ for the electrons and $\mu_{\text {gas }}=0.61$ for the gas. By directly integrating the electronic density profile up to a radius $R$, we obtain the gas mass enclosed within $R$,

$M_{\text {gas }}(R)=4 \pi \int_{0}^{R} \mu_{\mathrm{e}} m_{\mathrm{p}} n_{\mathrm{e}}(r) r^{2} \mathrm{~d} r$.

It is straightforward to deduce the gas fraction profile, defined as the ratio at a given radius between the gas mass and the total mass enclosed within $r$, as

$M_{\mathrm{gas}}(r)=f_{\mathrm{gas}}(r) M_{\mathrm{tot}}(r)$.
Finally, the total mass is directly related to $R_{\Delta}$ from its definition as $M_{\text {tot }}\left(r_{\Delta}\right)=\frac{4}{3} \pi \rho_{\mathrm{c}}(z) \Delta r_{\Delta}^{3}$, where $\rho_{\mathrm{c}}(z)$ is the critical density of the Universe at redshift $z$. Combining the value of $R_{\Delta}$ and $r_{\mathrm{p}}$, directly related to the pressure profile, we can therefore measure the concentration parameter $c_{\Delta}$.

\subsection{Extra datasets}

In addition to the NIKA data we consider the ACCEPT and Planck datasets.

\subsubsection{ACCEPT density profile}

We make use of the ACCEPT catalog (Archive of Chandra Cluster Entropy Profile Tables ${ }^{2}$, Cavagnolo et al. 2009). We only consider the deprojected X-ray density profile of CL J1226.9+3332, which is computed from the publicly available Chandra data. The angular resolution of Chandra, 0.5 arc$\mathrm{sec}$, is negligible compared to that of NIKA. As fully explained in Cavagnolo et al. (2009), the flux measured in the energy range $0.7-2.0 \mathrm{keV}$ is a good diagnosis of the ICM density (Eq. (6)). The high angular resolution surface brightness profile is therefore converted into a deprojected electron density profile using normalization and count rates taken from the spectral analysis. The profile extends up to $835 \mathrm{kpc}$, which corresponds approximately to $R_{500}$ (see Sect. 4).

\subsubsection{Planck integrated compton parameter}

The cluster CL J1226.9+3332 is not in the Planck tSZ cluster catalog (Planck Collaboration XXIX 2014) since its flux is diluted by the Planck beam, and it is therefore not detected with high enough signal-to-noise. Nevertheless, in addition to NIKA tSZ observations, we use the Planck maps to produce a Compton $y$ parameter map as described in Planck Collaboration XXI (2014; see Hurier et al. 2013 for the method). Its angular resolution is 7.5 arcmin, limited by the lowest Planck frequency channel used to construct it. This map is used to measure the integrated Compton parameter of CL J1226.9+3332 within $\theta_{\max }$, defined as

$Y_{\theta_{\max }}=\int_{\Omega\left(\theta_{\max }\right)} y \mathrm{~d} \Omega$.

The uncertainty on this quantity is obtained by applying the same integration on the map at positions around the cluster, where the noise is homogeneous and the map is free of emission. We also check on the jackknife (half-ring halfdifference, see Planck Collaboration I 2014) map that the error is consistent with the expected noise. We obtain $Y_{\Omega\left(15^{\prime}\right)}=$ $(0.94 \pm 0.36) \times 10^{-3} \operatorname{arcmin}^{2}$.

\subsection{Maximum likelihood analysis}

We aim at recovering the three-dimensional electronic pressure and electronic density profiles of CL J1226.9+3332. To do so, we use an approach in which input models are processed similarly to the measured tSZ signal such that they can be compared to it. Best-fit values of the electronic pressure and density model parameters are jointly obtained from a Markov Chain Monte Carlo (MCMC) approach, using a Metropolis-Hasting algorithm (Chib \& Greenberg 1995). A set of chains of tested models samples the multidimensional likelihood parameter space. At each step of the chains, a model map is computed by integrating the

2 http://www.pa.msu.edu/astro/MC2/accept/ 
tested pressure model along the line of sight. The map is then convolved with the NIKA beam and the pipeline transfer function. The model is converted into surface brightness using the $\mathrm{Jy} /$ beam to $y$ conversion factors given in Sect. 2.2. A radial temperature model is inferred from the pressure and the density models, and used to account for relativistic corrections (Itoh et al. 1998) on the tSZ map model. We also use the Planck integrated flux to add an extra constraint on the overall flux. The flux and the position of PS260 are simultaneously fitted. We only impose a Gaussian prior on its position based on the fit at $260 \mathrm{GHz}$, assuming a 3 arcsec uncertainty. We include a set of nuisance parameters in the fit, such as calibration uncertainties, map zero level, and the pointing position, which are randomly sampled within their error bars at each step.

The $\chi^{2}$ used in the Metropolis-Hasting acceptance (or rejection) process of the chain samples is defined as

$$
\begin{aligned}
\chi^{2}= & \chi_{\text {NIKA }}^{2}+\chi_{\text {ACCEPT }}^{2}+\chi_{\text {Planck }}^{2} \\
= & \sum_{i=1}^{N_{\text {pix }}}\left(\frac{M_{i}^{\text {NIKA }}-M_{i}^{\text {model }}}{\sigma_{i}^{\text {NIKA }}}\right)^{2} \\
& +\sum_{j=1}^{N_{\text {bin }}}\left(\frac{n_{\mathrm{e}}\left(r_{j}\right)^{\mathrm{ACCEPT}}-n_{\mathrm{e}}\left(r_{j}\right)^{\text {model }}}{\sigma_{j}^{\text {ACCEPT }}}\right)^{2} \\
& +\left(\frac{Y_{\theta_{\max }^{\text {Planck }}}^{\text {ACP }}-Y_{\theta_{\max }}^{\operatorname{model}}}{\sigma^{\text {Planck }}}\right)^{2}
\end{aligned}
$$

where the sums are made over the number of pixels in the NIKA map $\left(N_{\text {pix }}\right)$ and the number of radial bins of the ACCEPT density profile $\left(N_{\text {bin }}\right)$. The quantity $M$ represents the $150 \mathrm{GHz}$ only tSZ surface-brightness-plus-point-source map. The parameters $\sigma^{\mathrm{NIKA}}, \sigma^{\mathrm{ACCEPT}}$ and $\sigma^{\text {Planck }}$ are the respective errors, assumed to be Gaussian. While X-ray counts follow Poisson statistics, the deprojected density profile is computed by combining a set of random variables; therefore, assuming that the central limit theorem applies in this process, we expect the error statistics followed by the ACCEPT deprojected density profile to be Gaussian. It is also naturally the case for Planck and NIKA errors.

The convergence of the MCMC is ensured by the Gelman \& Rubin (1992) test. Once reached, the histogram of the chains along the considered parameter is marginalized over all other dimensions (including nuisance parameters) providing the posterior probability distribution for each fitted parameter. The integrated posterior probability distribution up to $68 \%$ probability gives the quoted errors.

To estimate the uncertainties on the derived cluster physical properties for each radial bin, we fully propagate the information contained in the MCMC parameter chains to the given quantity. For each set of parameters tested against the data (i.e., a model), we compute all derived physical quantities as a function of the radial distance. Therefore, for each radius, we obtain a probability distribution function for the considered quantity. We then compute the reference value of the given quantity as the median of the distribution and its error by integrating the distributions up to the requested confidence limit, for each radial bin.

\section{Results and discussions}

\subsection{NIKA dual-band detection and mapping of the tSZ signal}

From the MCMC analysis we obtain a flux of $1.9 \pm 0.2$ (stat.) $\mathrm{mJy}$ at $150 \mathrm{GHz}$ for PS260. With these results, PS260

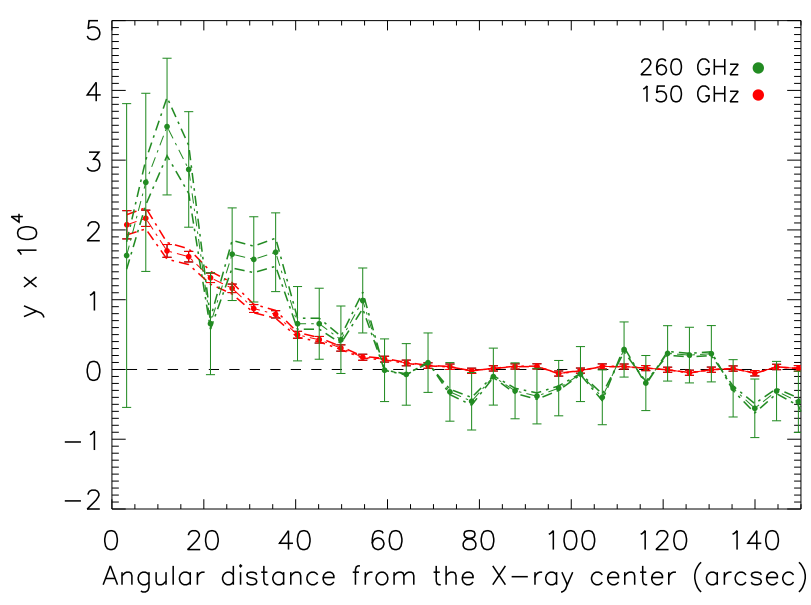

Fig. 4. Compton parameter profile $y$ of CL J1226.9+3332 at $150 \mathrm{GHz}$ (red) and $260 \mathrm{GHz}$ (green). The point source has been subtracted before extracting the profile. Statistical uncertainties are shown as error bars, and systematic uncertainties are given as a dashed-line envelope.

is subtracted from the maps in the following analysis. The Compton parameter profile is computed by averaging the signal within radial bins and accounting for the conversion between flux density and Compton parameter. Figure 4 shows the Compton parameter radial profile, computed from the X-ray center for both 150 and $260 \mathrm{GHz}$. The signal is detected on the profile up to about 1 arcmin at $150 \mathrm{GHz}$. Error bars are only statistical uncertainties; calibration uncertainties would result in an overall multiplicative factor to apply to the entire profile. The two profiles are compatible over the whole radial range. Nevertheless, we notice the presence of a few distinct peaks in the $260 \mathrm{GHz}$ profile. These peaks might indicate the presence of additional contamination from submillimeter sources, which are most probably below the noise in the $260 \mathrm{GHz}$ map. Assuming a dust-like spectrum, we expect the contribution from these sources to be negligible at $150 \mathrm{GHz}$. By fitting the $260 \mathrm{GHz}$ profile to the $150 \mathrm{GHz}$ one, taken as the model, we obtain a $7 \sigma$ tSZ detection at $260 \mathrm{GHz}$. We obtain a reduced $\chi^{2}$ of 0.95 with 24 degrees of freedom.

Figure 5 provides the raw, best-fit, point source subtracted and residual maps obtained from the maximum likelihood analysis. After subtracting PS260 the cluster appears circular at the NIKA resolution and is aligned with the X-ray peak on which the maps are centered. The signal is extended and clearly detected at the map level up to 1 arcmin scales. The NIKA map is morphologically consistent with previous interferometric observations by SZA (Joy et al. 2001; Muchovej et al. 2007; Mroczkowski et al. 2009) and does not show any evidence of being disturbed on large angular scales. However, the cluster core is slightly elongated toward the southwest at scales close to our our beam (and smaller). Using MUSTANG $90 \mathrm{GHz}$ observations, at an effective resolution of 11 arcsec, Korngut et al. (2011) have indeed detected a narrow ridge $\sim 20$ arcsec long located about 10 arcsec from the X-ray center towards the southwest. This is consistent with the hotter region found by Maughan et al. (2007) using Chandra and XMM X-ray data. Additional lensing observations from HST (Jee \& Tyson 2009) also reveals the presence of a secondary peak in the surface mass distribution in this region.

The NIKA observations agree with CL J1226.9+3332 being relaxed on large scales with a disturbed core, the origin of the latter being probably due to the merger of a smaller subcluster. Since NIKA probes scales between $\sim 20$ arcsec to a few arcmin, these observations complement the ones by MUSTANG 

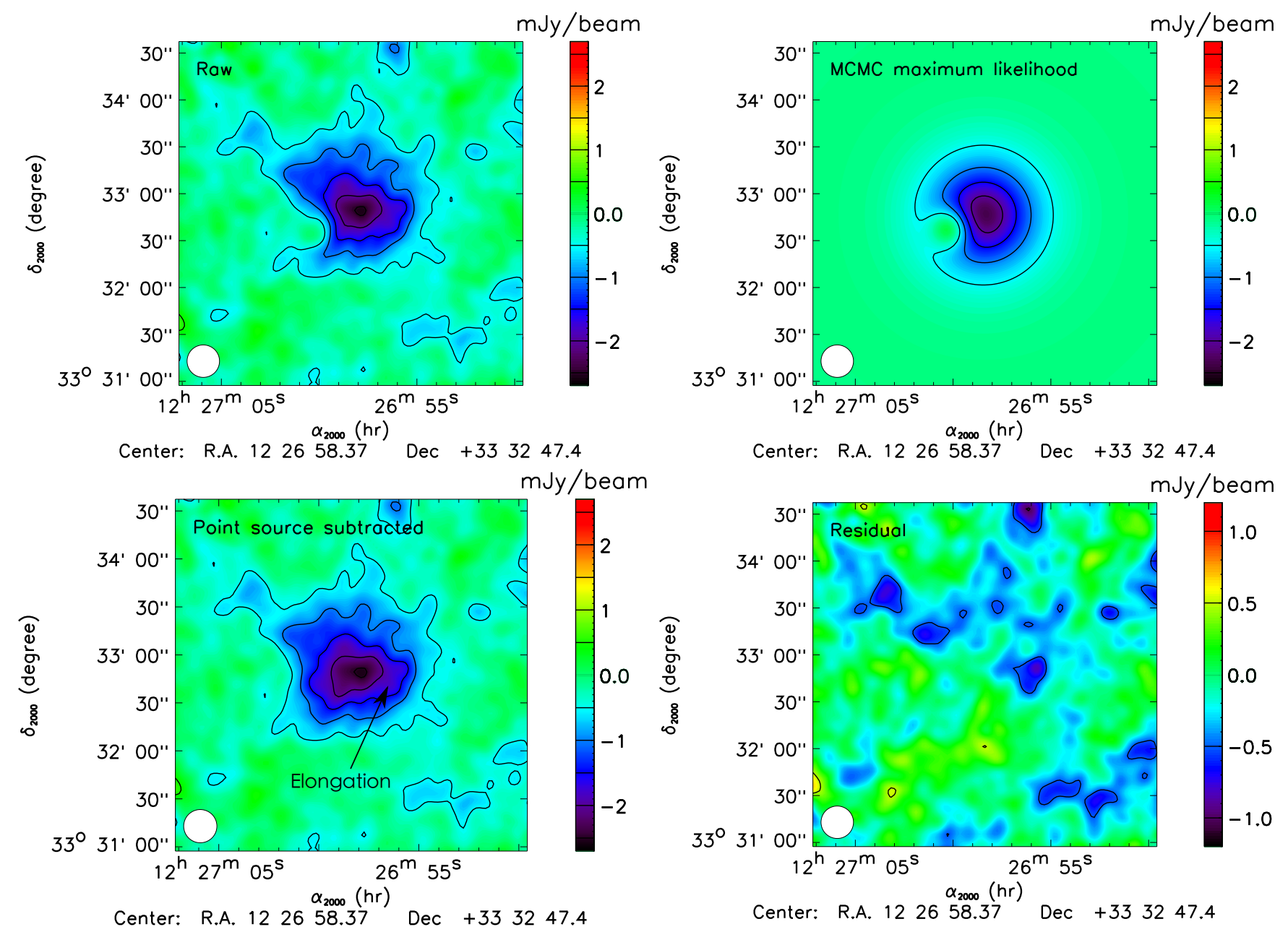

Fig. 5. Top left: NIKA $150 \mathrm{GHz}$ raw map of CL J1226.9+3332. Top right: MCMC maximum likelihood tSZ + point source model. Bottom left: point-source-subtracted map. Bottom right: tSZ + point-source-subtracted residual map. The contours are spaced by $0.5 \mathrm{mJy} / \mathrm{beam}$, and the maps have been smoothed with a 10 arcsec Gaussian filter. The effective beam is shown in the bottom left corner of each map.

on small scales $(\sim 10-50$ arcsec) and by SZA interferometric data that are the most sensitive on scales of a few arcmin. Finally, we notice that the NIKA residual map is well correlated with the temperature map presented in Maughan et al. (2007), i.e. the tSZ signal appears to be slightly stronger on the north (being under estimated by our spherically symmetric model), where the gas is hotter than it is in the south (respectively, overestimated), where the gas is cooler.

By measuring the integrated flux toward the cluster within a 50 arcsec radius circle centered on the X-ray peak at both NIKA wavelengths, it is possible to set constraints on the kSZ contribution (see Sect. 3.1.2). As shown in Fig. 6, the kSZ spectrum amplitude is compatible with zero within $1 \sigma$. Assuming the cluster average temperature within the region considered to be $T_{\mathrm{e}}=10 \pm 1 \mathrm{keV}$, we infer a limit on the line-of-sight velocity of $v_{z}=-445 \pm 461 \mathrm{~km} \mathrm{~s}^{-1}$ at $1 \sigma$ including calibration uncertainties.

\subsection{Intracluster medium radial distribution}

The best-fit density profile is represented in Fig. 7 with the data points of the ACCEPT catalog used to fit it. As shown in the bottom residual profile ( $\chi$, the difference between data points and the best-fit model normalized by the data point errors), the model fits the data over the full radial range for the purpose

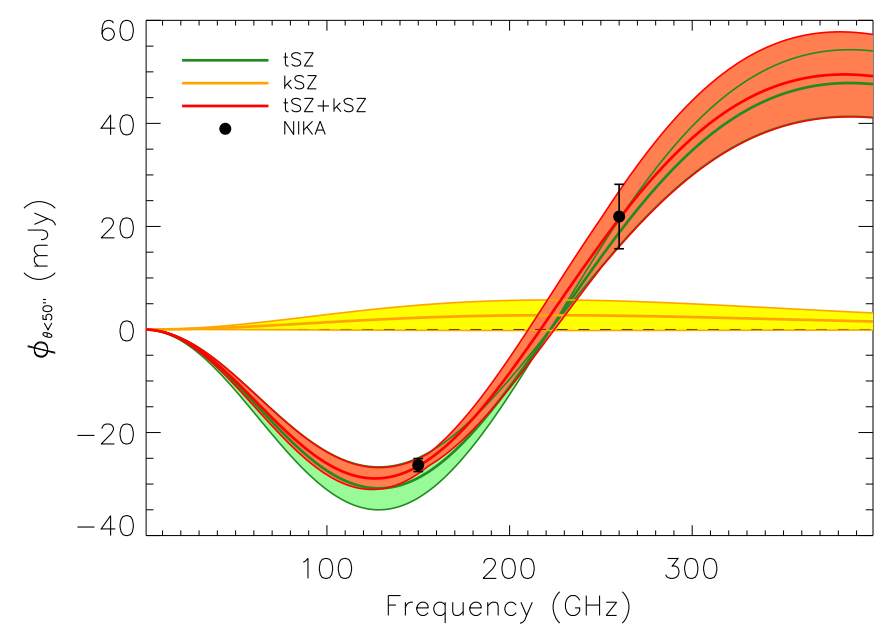

Fig. 6. Constraints on the kSZ and tSZ spectra computed within 50 arcsec of the X-ray center. The green, yellow, and red swaths give the tSZ, $\mathrm{kSZ}$, and $\mathrm{tSZ}+\mathrm{kSZ}$ contributions, respectively. The two data points are the NIKA measurements.

of this paper. Since the best-fit density profile depends on the choice of the pressure profile slope only through the relativistic correction (see Sect. 3.4), we show the result only for the first 


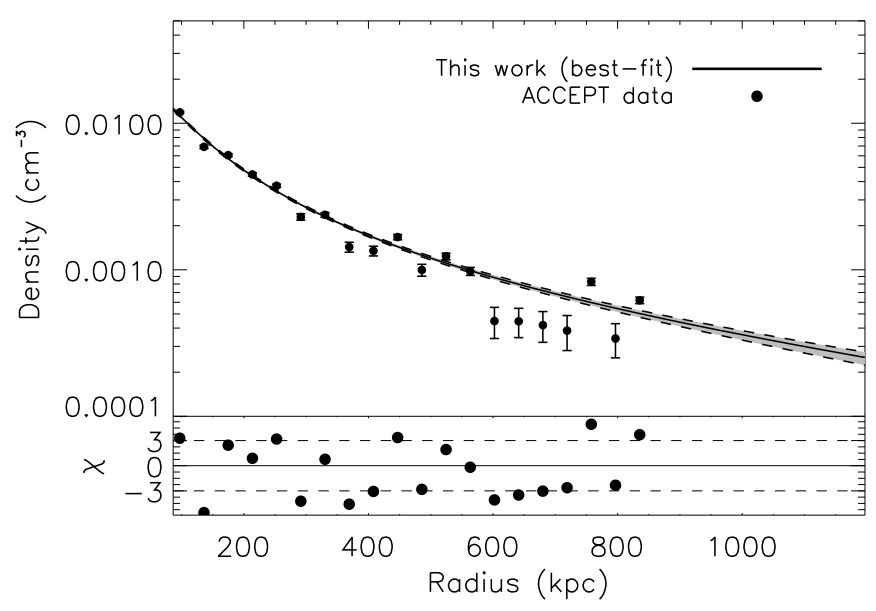

Fig. 7. Density profile as a function of physical distance from the cluster center. The data correspond to those from the ACCEPT database (Cavagnolo et al. 2009) discussed in the text. The solid line represents the best-fit density model. The $1 \sigma$ uncertainties are represented by the gray contours. The difference between the data and the best-fit model normalized by the $1 \sigma$ uncertainties $(\chi)$ is also shown.

case, our baseline PPC pressure profile, and the differences between models are insignificant.

In Fig. 8 we present the radial distributions of the pressure, temperature, and entropy of the ICM of CL J1226.9+3332 derived using NIKA data. Uncertainties are given at $68 \%$ confidence level and account for both statistical and NIKA overall calibration errors. The profiles corresponding to the different pressure profile models, PPC, NNN, and FPC (see Sect. 3.2.1) are given.

The pressure (left) is characterized well by NIKA, with less than $10 \%$ uncertainty below $500 \mathrm{kpc}$ and up to $25 \%$ at $1500 \mathrm{kpc}$ for PPC. The profile is best constrained around $250 \mathrm{kpc}$, corresponding to $\sim 30$ arcsec when projected onto the sky, where NIKA is most sensitive. The PPC profile is in qualitative agreement with the one obtained by Mroczkowski et al. (2009), despite a different choice of slope parameter. When using the same modeling, NNN, we find a good agreement, particularly on small scales. As expected, when loosening the constraints on parameter $a$, as in the case of the FPC profile, uncertainties increase by a factor of about 1.5 . Thanks to the degeneracy between the pressure profile parameters, the different models agree well in the region where NIKA is sensitive. At both larger and smaller scales, the models tend to deviate from one another up to more than $1 \sigma$, in particular NNN versus PPC and FPC, which directly propagates onto the other derived profiles, as discussed below.

The temperature profile, derived from the pressure and the density, presents a core value of about $15 \mathrm{keV}$ and decreases toward the outskirts of the cluster, reaching about $5 \mathrm{keV}$ around $1500 \mathrm{kpc}$. For the PPC model we find uncertainties of about $10-15 \%$. The profile is slightly higher, but compatible within errors to those measured by Mroczkowski et al. (2009) with SZA and Maughan et al. (2007) with a detailed X-ray (Chandra + $\mathrm{XMM}$ ) analysis. All three tested pressure profile models give compatible temperature results. Nevertheless, we notice that the core slope, $c$, obtained by Comis et al. (2011) tends to indicate a cooler core below $200 \mathrm{kpc}$, while it is not the case for NNN. This is because the core temperature, which is the ratio between the pressure and the density, is directly related to the core pressure slope $c$. The flatter core of PPC and FPC, with respect to
NNN, leads to a cooler core for the same density profile. Higher angular resolution $\mathrm{tSZ}$ observations would be necessary to provide constraints on the core slope pressure parameter $c$.

The entropy profile is generally described well by $K(r)=$ $K_{0}+K_{100}\left(\frac{r}{100 \mathrm{kpc}}\right)^{\alpha_{K}}$ (e.g., Pratt et al. 2010; Cavagnolo et al. 2009), where $K_{0}$ is called the core entropy, $K_{100}$ is a normalization, and $\alpha_{K}$ provides the slope of the profile. Large core entropies are expected for clusters with disturbed core such as CL J1226.9+3332. The obtained entropy profile (right), shown on a logarithmic scale for both axes, is described well by a simple power law in the range directly probed by NIKA $(\gtrsim 100 \mathrm{kpc})$. As we fixed the pressure profile parameter $c=0.014$ (Comis et al. 2011) in our baseline pressure model, which is expected to truly extrapolate the pressure parametrization on small scales, we expect the entropy profile to be accurate below $100 \mathrm{kpc}$ but limit ourselves to a qualitative discussion. The entropy profile shows signs of flattening below this scale with a core entropy above $100 \mathrm{keV} \mathrm{cm}{ }^{2}$, which would indicate that CL J1226.9+3332 is disturbed on small scales. At large radii, the slope seems to change but the error bars are too large for this effect to be measured. This discussion is also valid in the case of the NNN model, even if the core entropy tends to deviate by more than $1 \sigma$ between the two. Our baseline model, PPC, is fully compatible with the one obtained by Maughan et al. (2007). At large radii, however, the NNN profile is in better agreement with this X-ray analysis, with the main differences coming from the choice of the slope parameters at the core and the outer radii of the pressure profile.

The total mass and the gas fraction profiles are presented in Fig. 9. From the total mass profile we extract $R_{500}=930_{-43}^{+50} \mathrm{kpc}$, which in turn gives $M_{500}=5.96_{-0.79}^{+1.02} \times 10^{14} M_{\odot}$, compatible with previous measurements (e.g., Mroczkowski et al. 2009; Maughan et al. 2007). We obtain a gas fraction within $R_{500}$ of $f_{\text {gas }}\left(R_{500}\right)=0.146_{-0.030}^{+0.041}$. The total mass PPC and NNN profiles give compatible results over the full radial range. Small differences between the two are most noticeable in the range where NIKA is not very sensitive, i.e., on scales below $100 \mathrm{kpc}$ where NNN is higher than PPC, mainly due to the difference in the pressure profile. The FPC model is fully compatible with the two other ones, and it presents larger error contours. The results are similar for the gas fraction, for which PPC presents a flattening below $200 \mathrm{kpc}$, while NNN keeps decreasing. Assuming the gas fraction of CL J1226.9+3332 within $R_{500}$ to be a good representation of the matter content in the Universe, we compare it to its expected gas fraction using Planck Collaboration XVI (2014) cold dark matter, $\Omega_{\mathrm{c}}$, and baryon density, $\Omega_{\mathrm{b}}$, as $f_{\mathrm{gas}}=\frac{\Omega_{\mathrm{b}}}{\Omega_{\mathrm{c}}+\Omega_{\mathrm{b}}}=$ 0.156 . We find that it is compatible with our result within error bars.

The posterior ICM distribution is compatible in all cases with the integrated tSZ flux measured by Planck. The main outcomes of our analysis are summarized in Table 3.

\subsection{J1226.9+3332 and the tSZ-Mass scaling relations}

Clusters of galaxies are usually used for cosmological studies assuming a self-similar scenario because they are expected to be a scaled version of one another. In practice, non-gravitational processes can induce dispersion in the general trend and biases. The Planck satellite has recently released the largest tSZ selected cluster sample (1227 objects, Planck Collaboration XXIX 2014). To use this catalog for cosmology, Planck Collaboration XX (2014) have calibrated the 

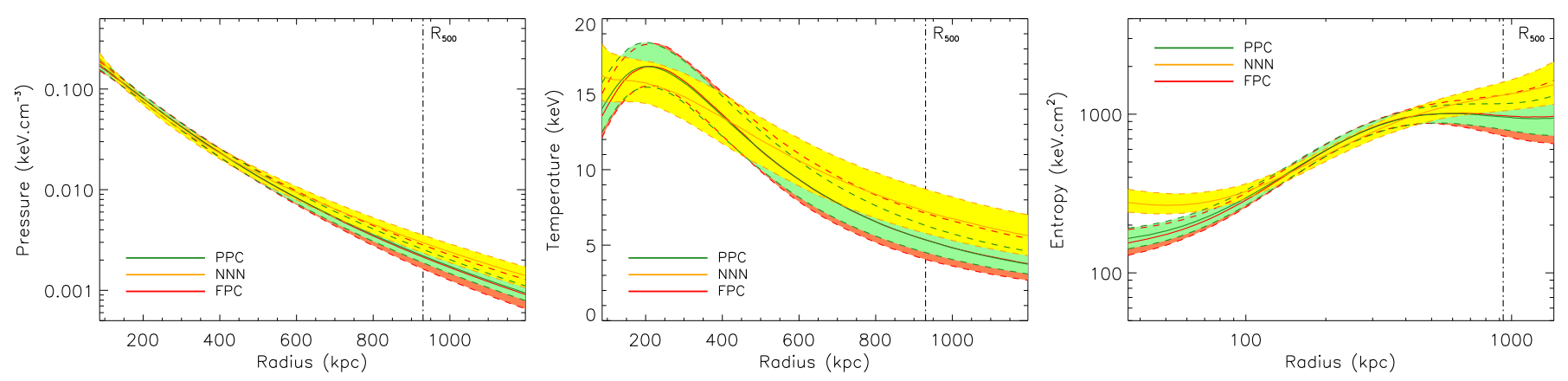

Fig. 8. Pressure (left), temperature (middle) and entropy (right) profiles as a function of physical distance from the cluster center. The green, yellow, and red swaths provide the $68 \%$ confidence limit, accounting for both calibration and statistical uncertainties. They correspond to modeling of the pressure profile with different choices for slope parameters $(a, b, c)$ as written in the legend. Once projected, a distance of $500 \mathrm{kpc}$ corresponds to about 1 arcmin at the cluster redshift.
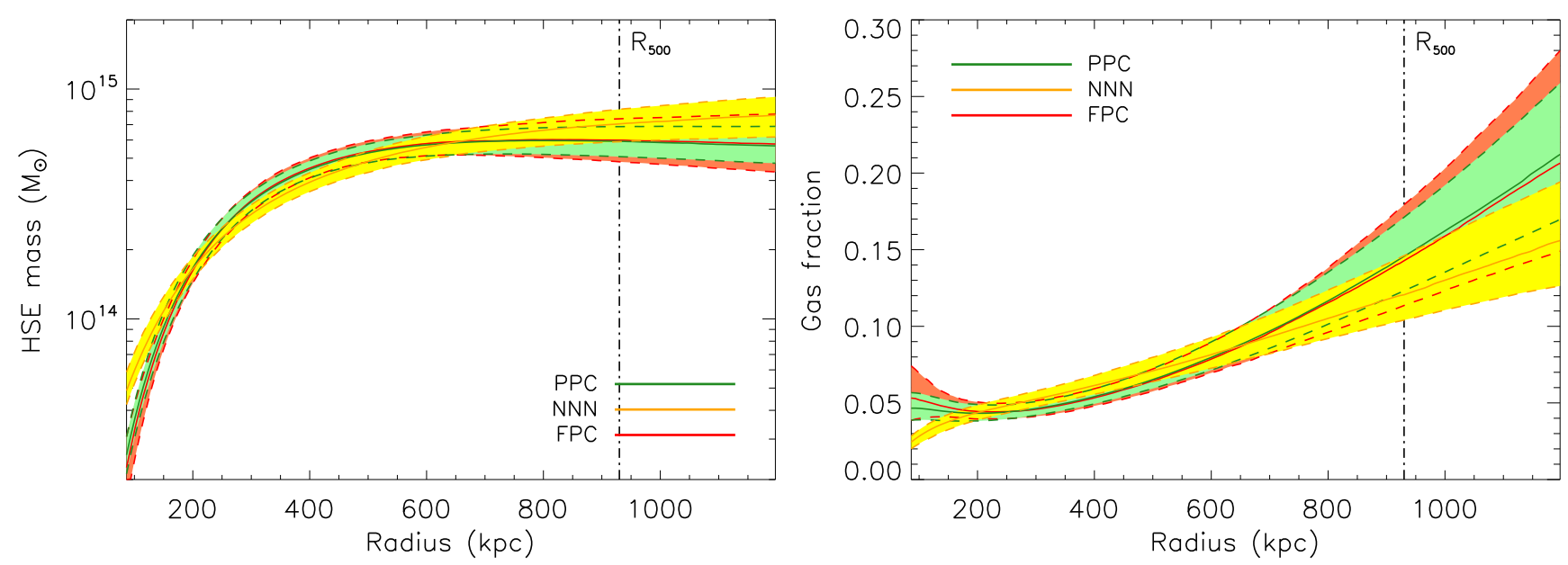

Fig. 9. NIKA best-fit derived radial profiles for hydrostatic equilibrium (HSE) total mass (left) and the gas fraction (right). The color code is the same as in Fig. 8.

relation between $Y_{\theta_{500}} \equiv Y_{500}$ and $M_{500}$

$E(z)^{-2 / 3}\left[\frac{D_{\mathrm{A}}^{2} Y_{500}}{10^{-4} \mathrm{Mpc}^{2}}\right]=10^{-0.19 \pm 0.02}\left[\frac{(1-b) M_{500}}{6 \times 10^{14} M_{\odot}}\right]^{1.79 \pm 0.08}$,

where $E(z)=\sqrt{(1+z)^{3} \Omega_{\mathrm{M}}+\Omega_{\Lambda}}$. The extra bias term, $(1-b)$, corresponds to the expectation that the hydrostatic equilibrium (HSE) mass underestimates the true mass due to non-thermal pressure, so that $M_{500}^{\mathrm{HSE}}=(1-b) M_{500}$. In this paper we set $b=$ 0.2 , because it was the baseline for Planck Collaboration XX (2014).

As a demonstration of the potential of future NIKA2 tSZ dedicated large programs, we present a comparison of the recovered characteristics for CL J1226.9+3332 in terms of pressure profile and tSZ-mass scaling relation, to the Planck 2013 results (Planck Collaboration Int. V 2013; Planck Collaboration XX 2014). The left panel of Fig. 10 provides a comparison between the pressure profile of CL J1226.9+3332, at highredshift, and the average profile over 62 nearby clusters obtained by Planck Collaboration Int. V (2013). Both have been normalized to account for the mass and redshift dependance by $f(M)=\left(\frac{M_{500}}{3 \times 10^{14} M_{\odot}} \frac{H_{0}}{70 \mathrm{~km} \mathrm{~s}^{-1} \mathrm{Mpc}^{-1}}\right)^{0.12}$ as detailed in Planck Collaboration Int. V (2013). The NIKA data show that the normalized pressure profile of CL J1226.9+3332 is among the highest ones, but does not show any significant evidence of non-standard redshift evolution, within error bars. In addition we notice that our error bars are model dependent and do not reflect the full uncertainty of the data. The evolution of the pressure profile with redshift has been statistically tested recently using a Chandra X-ray analysis of 80 SPT clusters (McDonald et al. 2014) with a highest bin at a mean redshift $z=0.82$. They find no significant evolution, apart from the cluster's cores, and agree with a standard redshift evolution of the pressure distribution among clusters. In the right hand panel of Fig. 10, we present $Y_{500}$ as a function of $M_{500}$ for CL J1226.9+3332. For comparison we also show the best-fit Planck Collaboration XX (2014) scaling law and the data corresponding to the 71 clusters used for its calibration. The mean redshift of this cluster sample is 0.195 with a maximum redshift of 0.447 . The cluster CL J1226.9+3332, at $z=0.89$, is consistent with the Planck Collaboration XX (2014) scaling relation. This single data point does not allow us to draw any conclusion on the evolution with redshift. However, our results illustrate the strength of such measurements based on a sample of a few tens of clusters with future NIKA2 observations. This will indeed allow us to precisely constrain the redshift evolution of scaling relations, on the basis of individual measurements.

\section{Summary and conclusions}

The NIKA camera at the IRAM 30-m telescope was used to image the cluster of galaxies CL J1226.9+3332 via the tSZ effect at 

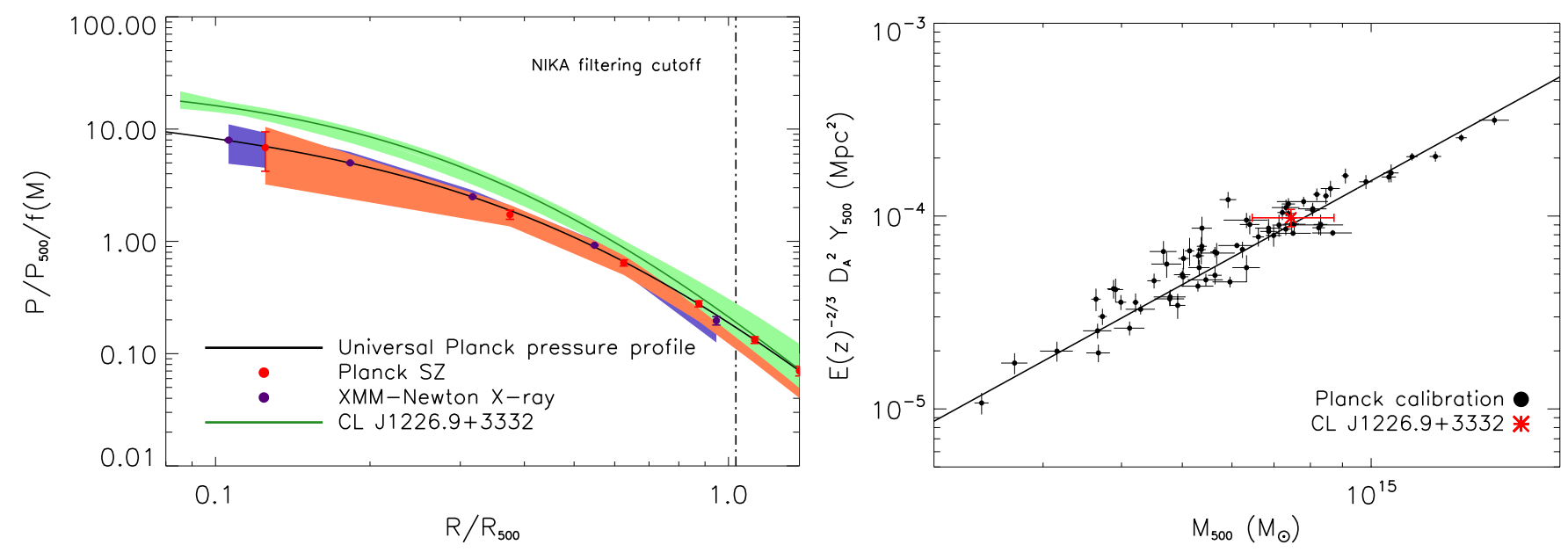

Fig. 10. Left: Planck universal pressure profile (black line), together with the best-fit profile obtained for CL J1226.9+3332 in green with the $1 \sigma$ error as a light green shadow accounting for all PPC, FPC, and NNN profile's. The Planck average of the individual pressure profiles across the 62 nearby cluster sample is given as red data points and the stacked pressure profile derived from the XMM data for the same sample is also given as purple dots (Planck Collaboration Int. V 2013). The dispersion about the respective tSZ and X-ray profiles are shown by shaded area with similar (lighter) colors. The scale corresponding to the one at which the NIKA data start to be affected by filtering is also given as a vertical dashed line. Right: Planck $Y_{500}-M_{500}$ calibration (Planck Collaboration XX 2014), together with the NIKA value obtained for CL J1226.9+3332 in the case of the PPC pressure profile parameterization. The Planck scaling law is represented as a black line, and the data points of the clusters used for its calibration are given as black dots. The NIKA data point is given by the red star.

Table 3. Main results of the MCMC analysis.

\begin{tabular}{|c|c|}
\hline PPC pressure profile & \\
\hline $\begin{array}{c}M_{500} \\
R_{500} \\
\theta_{500} \\
f_{\text {gas }}\left(R_{500}\right) \\
\quad Y_{500}\end{array}$ & $\begin{array}{c}5.96_{-0.79}^{+1.02} \times 10^{14} M_{\odot} \\
930^{+50} \mathrm{kpc} \\
1.93_{-0.09}^{+0.10} \mathrm{arcmin} \\
0.146_{-0.030}^{+0.041} \\
0.598_{-0.060}^{+0.063} \times 10^{-3} \mathrm{arcmin}^{2}\end{array}$ \\
\hline \multicolumn{2}{|l|}{ FPC pressure profile } \\
\hline $\begin{array}{c}M_{500} \\
R_{500} \\
\theta_{500} \\
f_{\text {gas }}\left(R_{500}\right) \\
Y_{500} \\
\end{array}$ & $\begin{array}{c}6.10_{-1.06}^{+1.52} \times 10^{14} M_{\odot} \\
937_{-58}^{+72} \mathrm{kpc} \\
1.95_{-0.12}^{+0.15} \mathrm{arcmin} \\
0.144_{-0.038}^{+0.062} \\
0.603_{-0.070}^{+0.098} \times 10^{-3} \mathrm{arcmin}^{2}\end{array}$ \\
\hline \multicolumn{2}{|l|}{ NNN pressure profile } \\
\hline $\begin{array}{c}M_{500} \\
R_{500} \\
\theta_{500} \\
f_{\text {gas }}\left(R_{500}\right) \\
Y_{500}\end{array}$ & $\begin{array}{c}7.30_{-1.34}^{+1.52} \times 10^{14} M_{\odot} \\
995^{+65} \mathrm{kpc} \\
2.07_{-0.13}^{+0.13} \mathrm{arcmin} \\
0.129_{-0.025}^{+0.041} \\
0.717_{-0.095}^{+0.117} \times 10^{-3} \mathrm{arcmin}^{2}\end{array}$ \\
\hline \multicolumn{2}{|l|}{ Point source PS260 } \\
\hline $\begin{array}{c}150 \mathrm{GHz} \text { flux } \\
260 \mathrm{GHz} \text { flux } \\
260 \mathrm{GHz} \text { best-fit position RA } \\
260 \mathrm{GHz} \text { best-fit position Dec }\end{array}$ & $\begin{array}{c}1.9 \pm 0.2 \text { (stat.) } \pm 0.1 \text { (cal.) mJy } \\
6.8 \pm 0.7 \text { (stat.) } \pm 1.0 \text { (cal.) mJy } \\
12 \mathrm{~h} 27 \mathrm{~m} 0.01 \mathrm{~s} \\
33^{\circ} 32^{\prime} 42.0^{\prime \prime}\end{array}$ \\
\hline
\end{tabular}

Notes. The quoted errors are given at $68 \%$ confidence level.

150 and $260 \mathrm{GHz}$ with 18.2 and 12.0 arcsec angular resolution, respectively. It provides the first resolved observation of this cluster at these frequencies. The cluster signal is detected in the two bands, but our tSZ analysis focuses on the $150 \mathrm{GHz}$ map since the signal-to-noise is higher at this frequency. A submillimeter point source is detected at $260 \mathrm{GHz}, 30$ arcsec away from the cluster center, showing the interest of the dual-band capabilities of NIKA to account for such contaminant. These observations, on scales 20-200 arcsec, complement previous single-dish $90 \mathrm{GHz}$ MUSTANG observations on scales in the range $\sim 10-50$ arcsec and interferometric SZA data at 30 and $90 \mathrm{GHz}$, which are the most sensitive at arcmin scales. The ICM morphology of the cluster agrees with these previous measurements. CL J1226.9+3332 appears relaxed on large scales and shows evidence of a disturbed core, most likely due to the merger of a subcluster to the southwest. It is also consistent with X-ray and lensing observations.

We used maximum likelihood analysis to constrain the pressure profile of the cluster via Markov Chain Monte Carlo sampling. The NIKA maps were combined with Chandra X-ray data using the ACCEPT data, to jointly derive ICM thermodynamic quantities (pressure, density, temperature, and entropy profiles). Planck tSZ data were also used to cross-check the overall flux of CL J1226.9+3332. The inferred temperature profile of the cluster exhibits a core value of about $15 \mathrm{keV}$ and decreases toward the outskirts, reaching about $5 \mathrm{keV}$ around $1 \mathrm{Mpc}$. The entropy profile is described well by a simple power law in the range probed by NIKA but shows sign of flattening below $100 \mathrm{kpc}$ with a core entropy above $100 \mathrm{keV} \mathrm{cm}^{2}$, agreeing with CL J1226.9+3332 being disturbed on small scales. Assuming that the hydrostatic equilibrium accurately applies to this cluster, we extracted the total mass and gas mass profile and derived the gas fraction profile. We measured $R_{500}=930_{-43}^{+50} \mathrm{kpc}$ and $M_{500}=5.96_{-0.79}^{+1.02} \times 10^{14} M_{\odot}$ at a $68 \%$ confidence level. We compared these results when assuming Planck tSZ-based pressureprofile slope parameters or X-ray/numerical simulation based ones and find that both choices give consistent results in general. These results are compatible within the uncertainties with previous tSZ and X-ray measurements.

NIKA is the prototype of NIKA2, which will be permanently installed at the IRAM 30-m telescope at the end of 2015. NIKA2 will contain about 5000 detectors, i.e., 15 times more than NIKA, within the same frequency bands and similar angular resolution. Its instantaneous field of view will accordingly increase from 1.8 to 6.5 arcmin. With such characteristics, 
NIKA2 will be well adapted to mapping the tSZ signal in intermediate and distant clusters of galaxies. The observation of CL J1226.9+3332 is part of a pilot study that aims at characterizing the possible scientific outcomes of large observing campaigns with NIKA2. Future NIKA2 dedicated tSZ observations of a few tens of clusters would allow study of the evolution of scaling and structural properties of clusters of galaxies out to $z \sim 1$. Here, by comparing our results to the expected tSZ-mass Planck scaling relations for a single cluster, we have shown that with more objects, NIKA2 will be able to calibrate the tSZ-mass scaling relation and its eventual redshift dependence.

Acknowledgements. We are thankful to the anonymous referee for useful comments that helped improve the quality of the paper. We gratefully thank Marian Douspis and the Planck collaboration for providing the Planck data points shown in the right hand panel of Fig. 10. We thank Marco De Petris for useful comments. We would like to thank the IRAM staff for their support during the campaign. This work has been partially funded by the Foundation Nanoscience Grenoble, the ANR under the contracts "MKIDS" and "NIKA". This work has been partially supported by the LabEx FOCUS ANR-11-LABX-0013. This work has benefited from the support of the European Research Council Advanced Grant ORISTARS under the European Union's Seventh Framework Program (Grant Agreement No. 291294). The NIKA dilution cryostat was designed and built at the Institut Néel. In particular, we acknowledge the crucial contribution of the Cryogenics Group and, in particular Gregory Garde, Henri Rodenas, Jean Paul Leggeri, and Philippe Camus. R.A. would like to thank the ENIGMASS French LabEx for funding this work. B.C. acknowledges support from the CNES post-doctoral fellowship program. E.P. acknowledges support from grant ANR11-BS56-015. A.R. would like to thank the FOCUS French LabEx for funding this work. A.R. acknowledges support from the CNES doctoral fellowship program.

\section{References}

Adam, R., Comis, B., Macías-Pérez, J. F., et al. 2014, A\&A, 569, A66 Allen, S. W., Evrard, A. E., \& Mantz, A. B. 2011, ARA\&A, 49, 409 Arnaud, M., Pratt, G. W., Piffaretti, R., et al. 2010, A\&A, 517, A92 Bartelmann, M. 2010, Class. Quant. Grav., 27, 233001 Birkinshaw, M. 1999, Phys. Rep., 310, 97

Bleem, L. E., Stalder, B., de Haan, T., et al. 2015, ApJS, 216, 27 Böhringer, H., \& Werner, N. 2010, A\&ARv., 18, 127

Bonamente, M., Joy, M. K., LaRoque, S. J., et al. 2006, ApJ, 647, 25 Bourrion, O., Bideaud, A., Benoit, A., et al. 2011, J. Inst., 6, P06012 Calvo, M., Roesch, M., Désert, F. X., et al. 2013, A\&A, 551, L12 Carlstrom, J. E., Holder, G. P., \& Reese, E. D. 2002, ARA\&A, 40, 643
Catalano, A., Calvo, M., Ponthieu, N., et al. 2014, A\&A, 569, A9

Cavagnolo, K. W., Donahue, M., Voit, G. M., \& Sun, M. 2009, ApJS, 182, 12 Cavaliere, A., \& Fusco-Femiano, R. 1978, A\&A, 70, 677

Chib, S., \& Greenberg, E. 1995, Am. Statistician, 49, 327

Comis, B., de Petris, M., Conte, A., Lamagna, L., \& de Gregori, S. 2011, MNRAS, 418, 1089

Ebeling, H., Jones, L. R., Fairley, B. W., et al. 2001, ApJ, 548, L23

Feretti, L., Giovannini, G., Govoni, F., \& Murgia, M. 2011, in IAU Symp., 274, eds. A. Bonanno, E. de Gouveia Dal Pino, \& A. G. Kosovichev, 340

Gelman, A., \& Rubin, D. B. 1992, Statistical Science, 7, 457

Hasselfield, M., Hilton, M., Marriage, T. A., et al. 2013, J. Cosmol. Astropart. Phys., 7, 8

Holden, B. P., Franx, M., Illingworth, G. D., et al. 2009, ApJ, 693, 617

Hurier, G., Macías-Pérez, J. F., \& Hildebrandt, S. 2013, A\&A, 558, A118

Itoh, N., Kohyama, Y., \& Nozawa, S. 1998, ApJ, 502, 7

Jee, M. J., \& Tyson, J. A. 2009, ApJ, 691, 1337

Joy, M., LaRoque, S., Grego, L., et al. 2001, ApJ, 551, L1

Kitayama, T. 2014, Prog. Theor. Exp. Phys., 06B111

Korngut, P. M., Dicker, S. R., Reese, E. D., et al. 2011, ApJ, 734, 10

Maughan, B. J., Jones, L. R., Ebeling, H., \& Scharf, C. 2004, MNRAS, 351, 1193

Maughan, B. J., Jones, C., Jones, L. R., \& Van Speybroeck, L. 2007, ApJ, 659, 1125

McDonald, M., Benson, B. A., Vikhlinin, A., et al. 2014, ApJ, 794, 67

Monfardini, A., Swenson, L. J., Bideaud, A., et al. 2010, A\&A, 521, A29

Monfardini, A., Benoit, A., Bideaud, A., et al. 2011, ApJS, 194, 24

Moreno, R. 2010, Neptune and Uranus planetary brightness temperature tabulation. Tech. Rep., ESA Herschel Science Center, available from ftp://ftp. sciops.esa.int/pub/hsc-calibration/PlanetaryModels/ESA2 Mroczkowski, T. 2011, ApJ, 728, L35

Mroczkowski, T., Bonamente, M., Carlstrom, J. E., et al. 2009, ApJ, 694, 1034 Muchovej, S., Mroczkowski, T., Carlstrom, J. E., et al. 2007, ApJ, 663, 708 Nagai, D. 2006, ApJ, 650, 538

Nagai, D., Kravtsov, A. V., \& Vikhlinin, A. 2007a, ApJ, 668, 1

Nagai, D., Vikhlinin, A., \& Kravtsov, A. V. 2007b, ApJ, 655, 98

Planck Collaboration Int. V. 2013, A\&A, 550, A131

Planck Collaboration I. 2014, A\&A, 571, A1

Planck Collaboration VIII. 2014, A\&A, 571, A8

Planck Collaboration XVI. 2014, A\&A, 571, A16

Planck Collaboration XX. 2014, A\&A, 571, A20

Planck Collaboration XXI. 2014, A\&A, 571, A21

Planck Collaboration XXIX. 2014, A\&A, 571, A29

Pratt, G. W., Arnaud, M., Piffaretti, R., et al. 2010, A\&A, 511, A85

Reichardt, C. L., Stalder, B., Bleem, L. E., et al. 2013, ApJ, 763, 127

Sarazin, C. L. 1988, S\&T, 76, 639

Sunyaev, R. A., \& Zel'dovich, Y. B. 1972, Astrophys. Space Phys. Res., 4, 173

Sunyaev, R. A., \& Zel'dovich, Y. B. 1980, ARA\&A, 18, 537

Vikhlinin, A., Kravtsov, A., Forman, W., et al. 2006, ApJ, 640, 691

Voit, G. M. 2005, Rev. Mod. Phys., 77, 207

Zwicky, F. 1933, Helvetica Phys. Acta, 6, 110 